NIST Special Publication 260-126 Rev 2013

\title{
The NIST Traceable Reference Material Program for Gas Standards
}

William D. Dorko (retired) Michael E. Kelley Franklin R. Guenther

This publication is available free of charge from: http://dx.doi.org/10.6028/NIST.SP.260-126rev2013

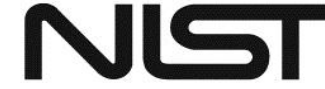

National Institute of Standards and Technology

U.S. Department of Commerce 


\title{
NIST Special Publication 260-126 Rev 2013
}

\section{The NIST Traceable Reference Material Program for Gas Standards}

\author{
William D. Dorko (retired) \\ Michael E. Kelley \\ Chemical Sciences Division \\ Materials Measurement Laboratory
}

This publication is available free of charge from: http://dx.doi.org/10.6028/NIST.SP.260-126rev2013

February 2015

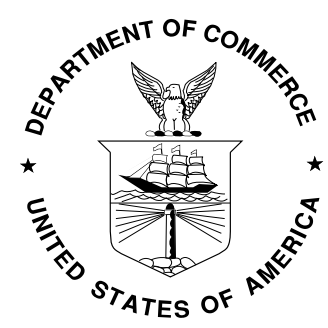

U.S. Department of Commerce Penny Pritzker, Secretary

National Institute of Standards and Technology Willie May, Acting Under Secretary of Commerce for Standards and Technology and Acting Director 
Certain commercial entities, equipment, or materials may be identified in this document in order to describe an experimental procedure or concept adequately. Such identification is not intended to imply recommendation or endorsement by the National Institute of Standards and Technology, nor is it intended to imply that the entities, materials, or equipment are necessarily the best available for the purpose.

National Institute of Standards and Technology Special Publication 260-126 Rev 2013

Natl. Inst. Stand. Technol. Spec. Publ. 260-126 Rev 2013, 41 pages (February 2015) CODEN: NSPUE2

This publication is available free of charge from: http://dx.doi.org/10.6028/NIST.SP.260-126rev2013 
(NIST Special Publication 260-126rev2013)

\title{
THE NIST TRACEABLE REFERENCE MATERIAL PROGRAM FOR GAS STANDARDS
}

\author{
by \\ William D. Dorko, Michael E. Kelley and Franklin R. Guenther \\ Chemical Sciences Division \\ Chemical Science and Technology Laboratory \\ National Institute of Standards and Technology \\ Gaithersburg, Maryland 20899-8393
}

REVISION 12-23-2013 


\begin{abstract}
A program is described by which the concentration of commercially produced gas mixtures may be related to gaseous primary standards maintained by the National Institute of Standards and Technology (NIST). The gaseous mixtures, referred to as gas mixture IST Traceable Reference Materials (NTRMs), must be similar in composition to NIST primary standards and the concentration of the certified component must be at, or bracketed by, primary standards. Although the NTRM gas mixtures are produced and distributed by commercial vendors, the concentration value assignment is made by NIST. The responsibilities of the producer and NIST are detailed along with recommended procedures the producer should follow during production and analysis of the mixtures. Procedures also are included for the maintenance of NTRM batches. Appendices are included for the preparation of NTRMs related to various Standard Reference Materials.
\end{abstract}




\section{FOREWORD}

The National Institute of Standards and Technology (NIST), formerly the National Bureau of Standards, was established by Congress in 1901 and charged with the responsibility for establishing a measurement foundation to facilitate both national and international commerce. This charge was purposely stated in broad terms to allow NIST the ability to develop its programs to respond to changing national needs and priorities.

The evolving linkage between the U.S. and world economy has resulted in an increased awareness of the need for comparability among chemical data used to assess feedstock and product quality and/or evaluate processes. The lack of comparability in chemical measurements is also a key factor in the high costs for healthcare, environmental assessment, and remediation activities.

As a part of its congressional mandate, NIST develops and maintains reference methods for analysis, and certifies and distributes Standard Reference Materials (SRMs). The NIST SRM Program distributes over 1300 different SRMs and has over 60,000 customers, with approximately $25 \%$ located outside the United States. The Documents of Certification contain certified results and their associated uncertainties, and are considered to be legal documents. All 1300 available SRMs are described in the Standard Reference Materials Catalog, Special Publication 260 and at web site www.nist.gov/srm.

The gas mixture NTRM program was established in 1992 in partnership with EPA and Specialty Gas Companies as a means for providing end-users with the wide variety of certified gas standards needed to implement the "Emissions Trading" provision of the 1990 Amendments to the Clean Air Act. In general, a NIST Traceable Reference Material is material produced by a commercial supplier with a well-defined traceability linkage to NIST. This linkage is established via criteria and protocols defined by NIST that are tailored to meet the needs of the metrological community to be served. Reference materials producers adhering to these requirements will be allowed to use the "NTRM" Certification Mark. Gas mixture NTRMs are produced and distributed by Specialty Gas Companies with NIST oversight of the production and involvement in the analysis, and can be developed for any analyte, concentration and balance gas combination for which a NIST primary standard suite or measurement system with defined uncertainty exists. Certified concentration values are assigned by NIST according to a protocol provided in this document.

Stephen A. Wise, Chief

Analytical Chemistry Division 


\section{Table of Contents}

1. INTRODUCTION

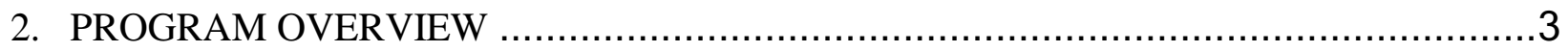

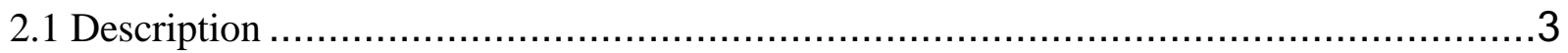

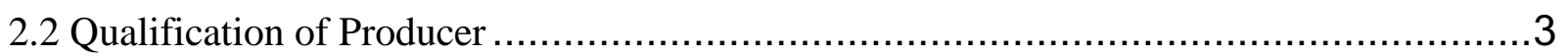

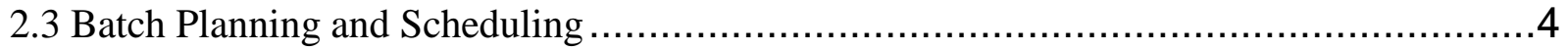

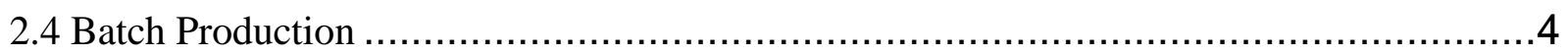

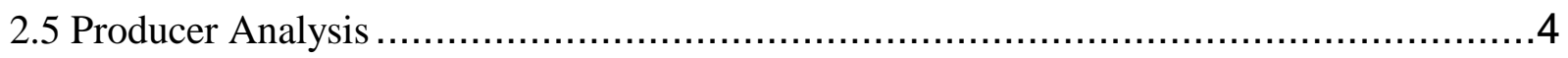

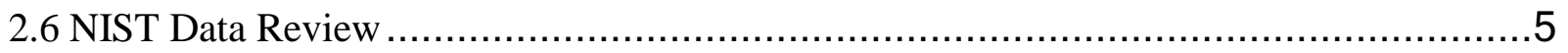

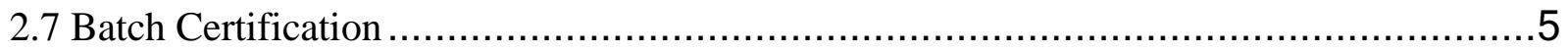

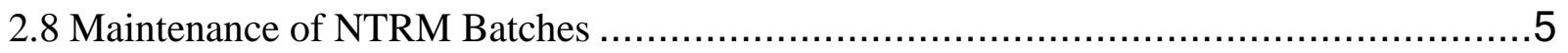

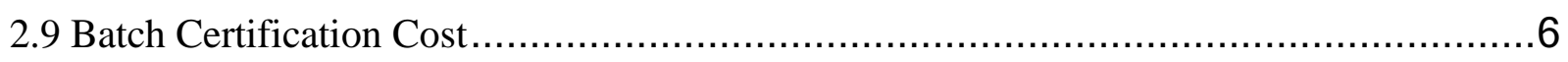

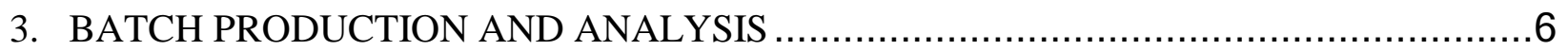

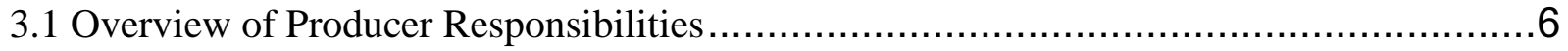

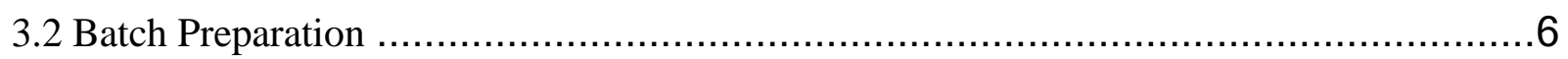

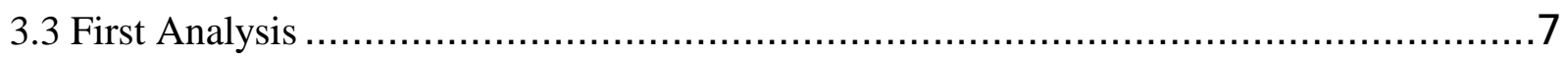

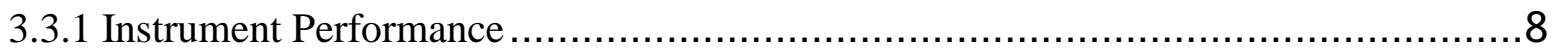

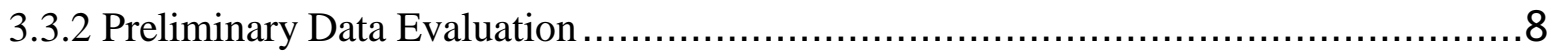

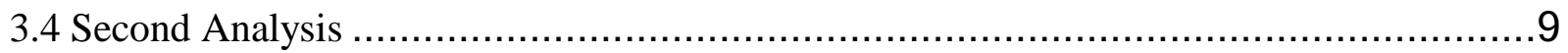

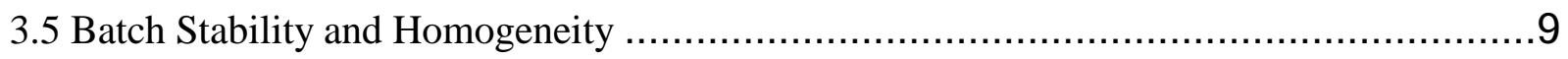

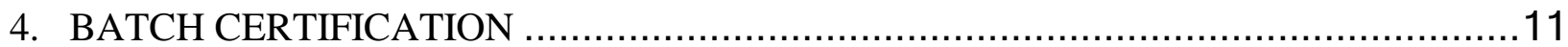

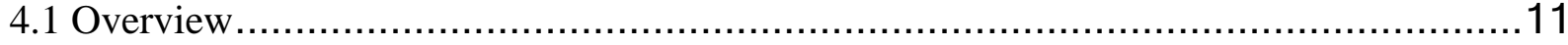

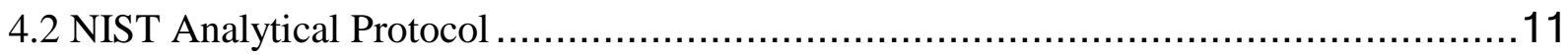

4.3 Producer's Data Validation and Batch Value Assignment ..................................... 12

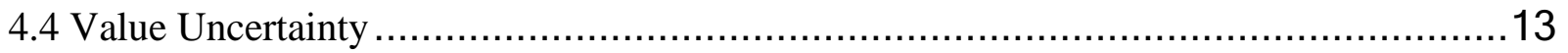

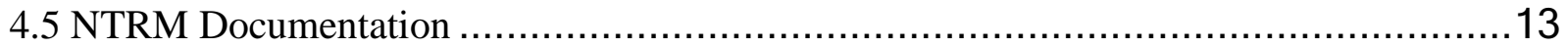

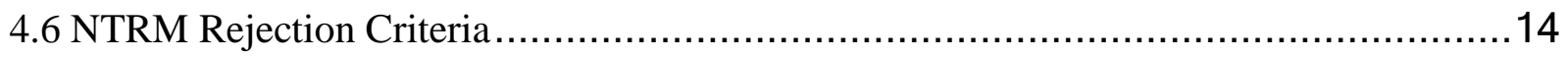

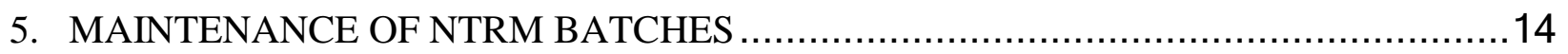

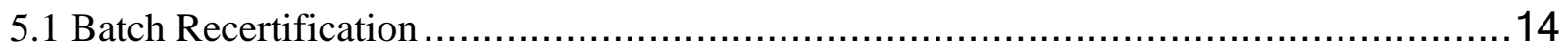

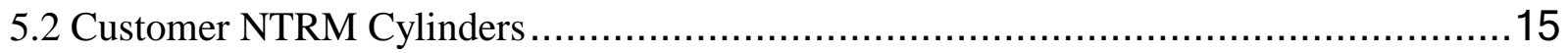

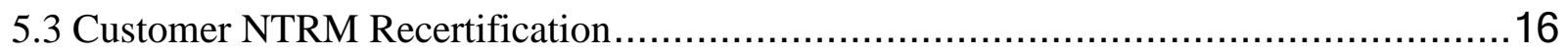

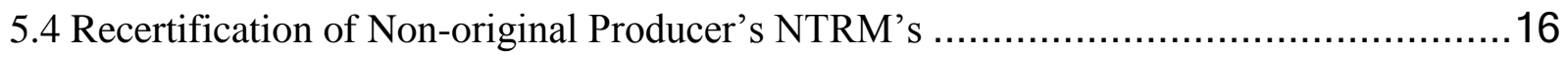

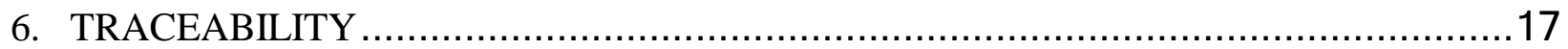

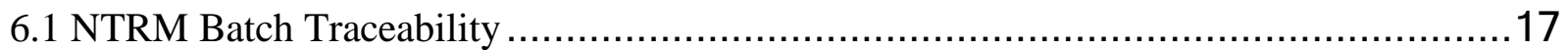

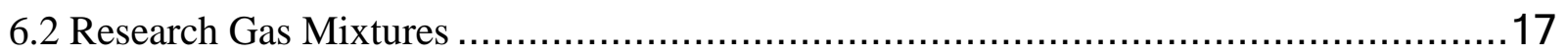

6.3 Mixtures Produced Referencing NTRMs and SRMs .........................................17 


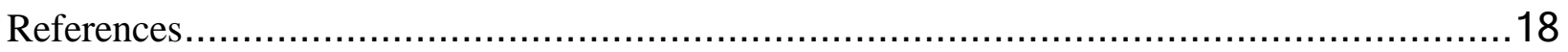

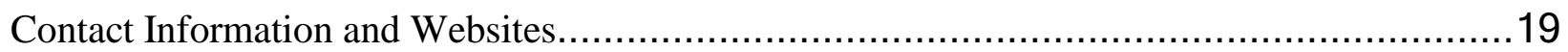

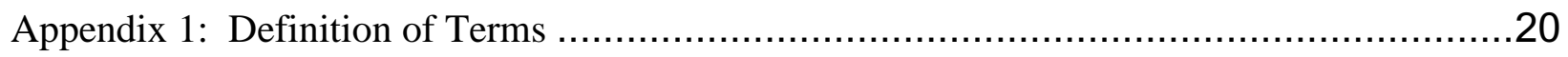

Appendix 2: Production and Certification Flow Chart with Referenced Sections .................23

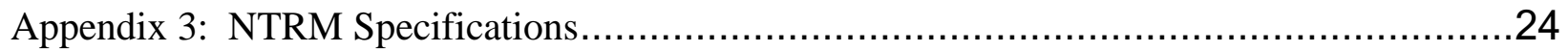

Appendix 4: Data Submission Forms for Certification and Recertification Data ................26

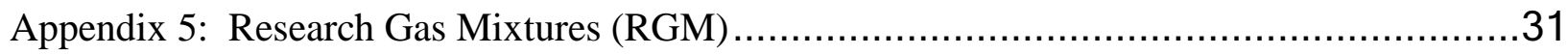

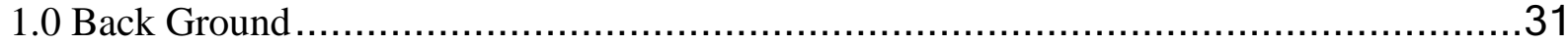

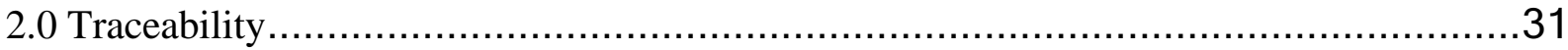

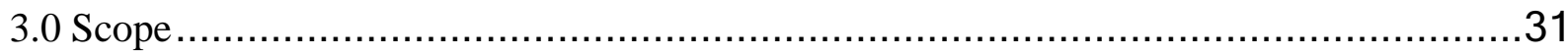




\section{INTRODUCTION}

A NIST Traceable Reference Material (NTRM) is a reference material produced by a commercial supplier with a well-defined traceability (the definition of emphasized words can be found in Appendix 1, "Definition of Terms") to the National Institute of Standards and Technology (NIST). This traceability is established via criteria and protocols defined by NIST that are tailored to meet the needs of the metrological community to be served. The NTRM concept was established to allow NIST to respond to the increasing needs for high quality reference materials with constant human and financial resources. Reference material producers adhering to these requirements are allowed to use the "NTRM" Certification Mark to identify their product.

The gas mixture NTRM program was established in 1992 in partnership with U.S. Environmental Protection Agency (EPA) and specialty gas companies as a means for providing end-users with the wide variety of certified gas standards needed to implement the "Emissions Trading" provision of the 1990 amendments to the Clean Air Act. Gas mixture NTRMs are produced and distributed by specialty gas companies with NIST oversight of the production and maintenance, and direct involvement in the analysis. NTRMs can be developed for any pollutant, concentration and balance gas combination for which a NIST primary standard or measurement system exists. The gas standards prepared according to this program are related, within known limits of uncertainty [1]), to specific gaseous primary standards or measurement system maintained by NIST.

This program is jointly administered by the NIST Analytical Chemistry Division and the NIST Measurement Services Division. The purpose of the program is to produce gas mixture NTRMs to supplement the supply of existing gaseous Standard Reference Materials (SRMs) and to be used where SRMs have been used in the past. The program is also designed to replace the EPA Certified Reference Material (CRM) program. The EPA has agreed to accept an NTRM in place of a CRM or SRM.

The procedures described herein are based on NIST experience relative to the production and certification of gaseous SRMs, and are intended to assure the development of reliable NTRMs. The integrity of the gas mixture NTRMs produced through this program is assured by NIST through active quality assurance measures. These measures include:

NIST oversight of the production and analysis of the NTRM by the producer

NIST analysis of cylinders representative of the NTRM

NIST assignment of the NTRM certified value and associated uncertainty

Documentation supplied by NIST

Details of the NTRM certification

Certificate of Traceability

Cylinder labels

NIST oversight of the long-term maintenance of the NTRM batch by the producer.

This document is being revised to include a number of updates and additions.

Updates:

1) Form for submission of certification data. The revision requests more information to be included to clarify blending and analytical facilities [appendix 4]. 
2) List of mixtures that can be supported as NTRMs. NIST continually updates its primary standards suites and measurement systems, therefore mixtures that could not be supported in the past can now be supported [appendix 3].

3) NIST Contact information. Addresses and personnel change; this revision provides the current information [section 1].

4) Recertification processes. Some NTRM producers have merged with other specialty gas companies and the manner by which the existing assumed NTRMs can be re-certified is addressed [section 5.4].

5) Certification periods. Experience with stability of both SRM and NTRM mixtures has allowed for the increase in some certification periods [section 4.5].

Additions:

1) NTRM Prime mixtures. Certain organizations require that mixtures that they use be analyzed at NIST and this is a mechanism by which this can be accomplished [section 2.1].

2) Research Gas Mixtures (RGMs). Defines a means of increasing the number of NIST standards to support the NTRM program and traceability in general [appendix 5].

3) Form for submission of recertifications data. No form was included in the original document and one has been developed and included to aide producers in providing adequate data to NIST for recertification [appendix 4].

4) List of websites and contact information

NIST Contact Information: gasntrm@nist.gov 


\section{PROGRAM OVERVIEW}

\subsection{Description}

This section gives an overview of the entire program (see Appendix 2 for flow chart). Where needed, subsequent sections explain various parts of the program in greater detail.

A gas mixture NTRM consists of a multi-cylinder batch prepared in such a manner that all the cylinders contain a gas mixture identical in composition and concentration. The cylinders, cylinder treatment, reagent gases, and gas blending procedure must assure mixture stability for a minimum period of time. The concentration of the NTRM must be within the bounds of a suite of gaseous NIST primary standards (Appendix 3) or measurement system [Appendix 1] with defined uncertainty. The concentration of an NTRM is determined by analysis with specific NIST standards. The uncertainty assigned to the concentration depends on the combined uncertainty in the NIST standards, the producer's homogeneity analysis, and the analysis performed at NIST. A single certified concentration and uncertainty value are assigned to the batch. The certified concentration and uncertainty, the period of time during which the concentration of the NTRM is certified, the presence of any impurities of consequence, and a list of all the cylinders included in the batch is given in a NIST Report of Analysis. A Certificate of Traceability is issued for each successful NTRM, along with labels to be affixed to each cylinder.

Because of special customer requirements, another type of NTRM is defined and this is the NTRM Prime. An NTRM Prime is prepared and analyzed in the same manner as the normal NTRM but the batch does not have to be homogeneous; however the individual cylinder mixtures must be stable. All cylinders are submitted to and analyzed by NIST, and each mixture is individually value and uncertainty assigned. This type NTRM was developed to fill the needs of certain end users that require a NIST analysis of each cylinder or for reactive mixtures where homogeneity is difficult.

Most of this document describes the normal NTRM process. Description of the NTRM Prime process will be specifically noted when applicable.

\subsection{Qualification of Producer}

In order to qualify for participation in the NTRM program, the producer must meet certain minimum requirements. These requirements are intended to assure the high quality of NTRMs and their availability to U.S. laboratories:

1) The producer must have in place a documented quality system. This should follow international or national guidelines (ISO 17025 or equivalent).

2) The producer must have the necessary facilities, equipment, and personnel to produce and analyze the gas mixture in the manner described in this document. NIST may require a meeting with key personnel and/or a site visit to the producer's facility. 
3) The producer must agree to one or more of the following:

a) make the NTRMs available for sale to U.S. customers;

b) demonstrate that traceable standards produced from NTRMs (as described in section 6.2) will be made available to U.S. customers; or,

c) demonstrate they will be used to produce gas standards according to the EPA protocol standards document [Ref. 2] for sale to US customers.

\subsection{Batch Planning and Scheduling}

Before an NTRM batch is prepared, the producer must contact NIST for discussions, including the determination of whether NIST has a measurement system to perform the work and the establishment of a production/certification schedule agreeable to both parties providing NTRM production is approved. At this time any questions can be addressed so that there is a mutual understanding of procedures. The producer must submit a plan describing the analytical procedures to be used, including the identification of the calibration standards. NIST will supply the producer with technical specifications the NTRM is expected to meet or exceed in order to be certified. After concurrence on pertinent points the batch preparation can proceed.

\subsection{Batch Production}

Each NTRM must be prepared as a multi-cylinder homogeneous batch (minimum 10 cylinders). The cylinders must be clean, of standard size and construction, and of known compatibility and history with the composition of the proposed NTRM. All NTRM cylinders within the batch must be identical with respect to size, construction, valve, and cylinder treatment. The cylinders must be equipped with valves of the appropriate material that conform to Compressed Gas Association (CGA) recommendations for the particular gas mixture. The reagent gases used must be of high purity and quality to ensure that impurity levels and mixture stability meets NIST technical specifications.

An NTRM Prime is blended in the same manner as a normal NTRM but the homogeneity requirements do not have to be met.

\subsection{Producer Analysis}

The responsibility of the producer is threefold:

1) to determine the concentration relationship among all of the cylinders in the batch (batch homogeneity);

2) to establish the average concentration of the batch; and,

3) to establish a baseline from which to compare future analyses performed to evaluate batch stability.

To accomplish this, one or more NIST certified standards are to be used. Standards equivalent to NIST certified standards may be used with prior approval of NIST. The producer's data are used to assess batch homogeneity and are included in the overall expanded uncertainty in the concentration of the batch. Thus 
it is imperative that the producer generates high quality data in order for the expanded uncertainty to be minimized. Section 3 provides more details on the analysis phase of NTRM batch production.

\subsection{NIST Data Review}

The producer must submit to NIST all the data generated from activities described in sections 2.4 and 2.5. The producer should review these data prior to submission to determine if the batch meets NIST specifications. Once the producer is confident that the batch meets specifications, the producer should submit the data electronically using a NIST approved data format. NIST will review the data to determine if the batch meets NIST specifications and to select representative cylinders from the batch for NIST evaluation. Upon request, the producer will provide NIST with any additional data and/or information relating to the production and analysis of the NTRM batch. Proprietary information is generally not needed for this review; however NIST can enter into nondisclosure agreements if necessary.

\subsection{Batch Certification}

After NIST reviews the data the producer must send the selected cylinders to NIST for evaluation. NIST will evaluate the cylinders for concentration and may analyze for impurities. The NIST analysis will involve comparisons of the NTRM cylinders to NIST standards or measurement system. NIST standards that may be used in these analyses include primary standards, SRMs, SRM lot standards and Working Standards. For convenience the term measurement system will be used in the rest of the document to refer to the NIST certification process. In general the analyses that NIST performs to value assign NTRM batches will be identical to those used in the certification of SRMs. The NTRM will be certified for a specific period of time dated from the NIST Report of Analysis. Non-reactive gas mixtures may have different certification periods than reactive gas mixtures.

At the end of the certification period the producer must submit recertification data to NIST in order for the certification period to be extended. As long as the producer maintains the batch as described in Section 5 , a customer purchasing an NTRM cylinder will have a certified period extending for the same length of time as the rest of the batch. More details on the NIST certification process can be found in Section 4.

\subsection{Maintenance of NTRM Batches}

It is the responsibility of the producer to maintain the NTRM batch over the potential lifetime of the individual cylinders. In general the batch standard is used to measure the stability of the entire batch. Periodic analysis of the batch standard against a NIST certified standard will allow a determination of the batch stability over time. NIST must be notified if the batch standard shows a change in concentration greater than half of the stated uncertainty for the batch. NIST will recommend a course of action to further assess batch stability. Actions that may be recommended include:

1) NIST reanalysis of the batch standard

2) analysis of some or all remaining batch cylinders by the producer; or,

3) recall of selected cylinders for analysis by the producer 
More details on the maintenance of NTRM batches can be found in Section 5.

\subsection{Batch Certification Cost}

NIST is compensated directly by the producer for the certification of each candidate NTRM batch. Fees are established on a yearly basis and are effective on October 1 of each year. Fees are based on the number of cylinders in the batch with an additional surcharge if the certification requires interpolation between primary standards. Also included in the fee is a charge for preparation of the Certificates of Traceability and the cylinder labels. A purchase order must be submitted to NIST before any work can proceed. Information regarding the location to which paper work or cylinders should be sent is obtained at the e-mail address: gasntrm@nist.gov. Purchase orders may be submitted by regular mail, FAX or e-mail. The organization's Taxpayer Identification Number (TIN) must be included.

\section{BATCH PRODUCTION AND ANALYSIS}

\subsection{Overview of Producer Responsibilities}

The NTRM batch must be blended in such a way as to assure gas mixture homogeneity and stability. After blending, the contents of each cylinder in the batch must be analyzed by the producer. The analytical procedure recommended here is considered to be the minimum effort necessary to adequately evaluate each batch for homogeneity and stability. All NTRMs must be stable, and each batch should be homogeneous in composition and concentration. An unstable batch must be rejected, while a batch exhibiting a small heterogeneity in concentration may be acceptable, providing that the resulting overall expanded uncertainty in the concentration does not exceed the desired level. The NTRM program is based on a batch certification; all cylinders comprising the batch are assigned the same absolute concentration. Thus, any batch heterogeneity will increase the uncertainty assigned to the certified value of the batch.

The objective of the producer's analysis is to compare each cylinder mixture (sample) in the batch to one or more NIST-certified standards. The simplest procedure would involve direct analysis of each NTRM sample with a NIST-certified standard, but with most analytical methods this approach would use a large proportion of the available gas. Consequently, it is recommended that the concentration be determined on a relative basis by selecting one sample from the batch, designated the batch standard (BS), to which all of the other samples are compared. The BS is then analyzed against one or more NIST certified standards. The concentration of all the other samples can be determined from the measured ratio to the batch standard.

Analyses for impurities which might compromise the use and stability of the NTRM will be performed using procedures determined by the producer. Maximum impurity levels are given in Appendix 3.

\subsection{Batch Preparation}

Before an NTRM batch is prepared, the producer must contact NIST for discussions, including the establishment of a production/certification schedule agreeable to both parties. At this time any questions can 
be addressed so that there is a mutual understanding of procedures. The producer must submit a plan describing the analytical procedures to be used, including the identification of the batch standard and calibration standards to be used. NIST will supply to the producer with technical specifications the NTRM is expected to meet or exceed in order to be certified. After concurrence on pertinent points the batch preparation can proceed.

Each NTRM must be prepared as a multi-cylinder homogeneous batch (minimum 10 cylinders). The cylinders which will contain the NTRM mixture must be clean and of known history and compatibility with the composition of the proposed NTRM. All cylinders must be identical with respect to size, construction, valve, and cylinder treatment. The cylinders must be equipped with valves of the appropriate material that conform to Compressed Gas Association (CGA) recommendations for the particular gas mixture.

Care must be exercised when filling the cylinders in order for the batch to meet the NIST homogeneity specifications. Any one of several fill methods can be used including:

1) filled from a single vessel containing a bulk mixture

2) filled from several containers connected together in such a manner that delivery occurs simultaneously from each container

3) filled by use of a dynamic blending system

4) filled by other methods shown to be effective by the producer.

The reagent gases must be of high purity and quality to ensure that impurity levels and mixture stability meets NIST specifications. The concentration of the component of interest (analyte) must be at, or bracketed by, NIST traceable standards (Appendix 1). A diagram of the filling manifold, including the position of each of the cylinders, must be maintained as part of the NTRM batch records. The identities of all cylinders will be reported to NIST referencing the cylinder number stamped permanently on the cylinders, along with a sequential sample number assigned to each cylinder. The sample numbers will span from one through the number of cylinders in the batch and will be permanently assigned to the cylinders throughout the valid certification periods of the NTRM.

Multicomponent NTRM mixtures may be produced as long as every component which is to be certified has a concentration at, or bracketed by, NIST traceable standards or other standards approved by NIST prior to use. Chemical interactions between components of the NTRM must not occur, or must have completed by the time the producer initiates the first analysis. The balance gas, cylinder walls, and valve must be chemically inert with respect to the certified components. Certification of each component is performed independently of all other components.

\subsection{First Analysis}

An initial analysis of the NTRM batch should be performed two or more days after preparation (see Appendix 3 for incubation period). One analytical protocol that may be used is to select one cylinder from the batch, designated the batch standard, to which all other samples from the batch are compared. The batch standard must be analyzed against appropriate SRM(s) or other NIST certified standards to determine concentration and to establish a reference for later analyses intended to confirm the stability of the batch. The BS should be analyzed at least six times using a NIST certified standard to calibrate the instrument. This 
analysis should consist of at least six alternations of the batch standard and the NIST standard. All of the remaining samples are then analyzed in duplicate, using the batch standard as a reference.

During the first analysis the producer must analyze for impurities using procedures.

NIST reserves the right to identify cylinders to be analyzed. For nitric oxide mixtures, all cylinders must be analyzed for nitrogen dioxide.

\subsubsection{Instrument Performance}

The general characteristics of the analyzer response relative to concentration must be known and demonstrated. This is accomplished by constructing a calibration curve using a minimum of three gas mixtures. The gas mixture nearest in concentration to the nominal batch concentration must be a NISTcertified standard. If the analyzer has a significantly nonlinear function then a minimum of four calibration standards are required. If a model using a straight line instead of a quadratic fit results in a predicted concentration different by $0.5 \%$ or more, then four or more standards and a quadratic fit must be used. The standards must include a NIST certified standard above and below the concentration of the NTRM (if such standards are available). Obviously this is not possible when the NTRM is being compared to either the lowest or highest concentration NIST-certified standard available in a particular analyte series; these situations will be addressed on a case-by-case basis considering the limits of NIST analytical capabilities.

Extrapolation beyond the calibration curve is not recommended without technical justification. Thus, the NTRM concentration should be adjusted to lie within the concentration bounds of the available calibration gases.

The principal elements that contribute to the imprecision of measurement include instrument sensitivity and drift. The sensitivity of the instrument should be high enough so that differences in concentration of $0.2 \%$ relative can be easily measured. Instruments are available that are capable of measuring all current SRMs with this sensitivity. However, most instruments exhibit a certain amount of signal drift. The drift has two components, short term drift (noise), and a long term drift characterized by a slowly changing signal under constant operating conditions. The effect of short term drift can be minimized by a number of techniques including multiple analysis of each sample, and signal averaging. The effect of long term drift can be minimized by frequent recalibration of the instrument. The characteristics of each analytical system should be considered individually to determine the optimum frequency of calibration and the approach taken to compensate for or control the effect of noise. It should be noted that the effort expended in minimizing the effects of drift will improve the precision of the analyses relating the NTRM to the NIST certified standard. In general, if duplicate analyses of single samples consistently agree within $0.2 \%$ relative, then the effect of noise is at an acceptable level, and if repeated analysis of the same sample performed at intervals during the analysis of a batch agree within the same limits, then the long term drift is at an acceptable level.

\subsubsection{Preliminary Data Evaluation}

Some preliminary conclusions regarding the homogeneity and stability of a batch may be drawn from the results of the first analysis. If the batch is homogeneous when filled, and if no subsequent reactions have occurred randomly in the cylinders, the concentration of all samples should be the same within the limits of 
precision of the analytical method. If heterogeneity among the samples is observed, it will not be possible at this time to determine whether such heterogeneity is due to improper preparation or to reactions in the cylinders.

If the first analysis indicates that no serious problem exists, the entire batch is set aside for 30 days. If the data indicate that the batch may be bad, NIST should be notified.

\subsection{Second Analysis}

After the required minimum 30-day hold period has passed, all samples in the batch must be analyzed in the same manner as the first analysis using the same NIST certified standard(s). The first analysis served to define the concentration of the NTRM and to test for batch homogeneity. Decisions regarding stability depend on the second analysis. Sufficient time will have elapsed so that the effects of slower reactions in individual cylinders can be measured through this subsequent analysis.

\subsection{Batch Stability and Homogeneity}

The stability and homogeneity of an NTRM batch must be carefully evaluated. The batch is considered stable if the concentration in each NTRM cylinder has not changed appreciably (less than the measurement uncertainty) between the first and second analyses. If no change is detected, the batch is most likely stable and the producer may proceed. However, if an inspection of the data from the first and second analyses reveals gross instability the batch must be discarded and remade. NIST will assist the producer in analyzing the data if there are questions concerning significance of the differences found.

If cylinder-to-cylinder differences within the batch are observed, then the producer and NIST must decide if this heterogeneity will result in an unacceptably large expanded uncertainty. An NTRM is useful only if the expanded uncertainty in the concentration is at or below a certain level. In general the expanded uncertainty should be less than two times the maximum stated uncertainty of the NIST certified standards used for calibration. If the heterogeneity is small enough then this level will not be exceeded. If it is large and the acceptable expanded uncertainty level will be exceeded, then the batch should be remade.

The concentration of the analyte(s) in all of the cylinders of an NTRM batch should be identical. Differences in concentration observed among cylinders should reflect only the combined uncertainty in the measurement process [1]. Differences greater than this combined uncertainty may arise from several sources. There can be differences that result from faulty mixing, or from dilution by residual gases left in the cylinder prior to transfer of the bulk mixture. A more critical source arises from reactions, chemical or physical, that occur in the cylinder after transfer. These are more critical because these reactions are largely due to wall effects, and because no two cylinder walls are alike, the reactions generally proceed at different rates in different cylinders. The two effects may be differentiated by sets of measurements made over a period of time sufficiently long so that the extent of reaction is greater than the measurement uncertainty in the analysis. If a batch is analyzed immediately after preparation and individual samples are found to have concentrations that vary more than the measurement uncertainty, then the mixture is either heterogeneous, or some or all of the samples in the batch are unstable. If the batch is reanalyzed after some time and each sample has essentially retained its previous value, then the samples are probably stable, but the batch is heterogeneous. If the 
concentration of one or more of the samples has changed significantly, then it is plausible to conclude that the batch is unstable.

It may not be necessary to discard a heterogeneous batch, but an unstable batch must be discarded. A heterogeneous but stable batch may be used if the heterogeneity is sufficiently small that the expanded uncertainty does not exceed the acceptable level. If during blending, several large cylinders are connected together to allow simultaneous delivery, then some heterogeneity in the batch may result if the concentrations in the source cylinders differ slightly. In the special case of large scale dynamic dilution systems where two components are mixed under constant flow conditions and are then compressed into the cylinders in the batch, some heterogeneity may result, depending on the design of the delivery system. If variations in concentration are observed in a batch prepared by this method, the variation may be related to the position of individual cylinders on the manifold, and it is therefore important to record the manifold position of each cylinder.

The nature of the concentration distribution of samples in a batch may give significant information concerning both the homogeneity and stability of the batch. A batch which has concentrations that vary no more than can be accounted for by the measurement uncertainty of the analytical method is probably homogeneous and stable. A batch in which the range of values exceeds significantly what might be expected on the basis of measurement uncertainty, and in which several samples are considerably below the average, is probably unstable. A batch in which the range of values is large but all concentrations are essentially symmetrically distributed around the average, was probably not prepared or transferred in such a way that homogeneity was assured. Occasionally, one or more samples in a batch will be found with concentrations appreciably different from the average for the batch. These may represent faults in the preparation or filling of the cylinder. These cylinders may be removed from the batch, provided the batch is otherwise shown to be stable and homogeneous.

All of the data at this stage of NTRM production must support the assumption that the batch is stable, homogeneous, and meets all other specifications for an NTRM. If there is any suspicion that the batch may be unstable or heterogeneous, then further analytical work should be conducted before a final decision is made to proceed.

At this point there should be sufficient data for the producer to decide whether or not the batch will qualify as an NTRM. This decision must be based on the measured concentration, stability and homogeneity. Ultimately the decision will be subject to an independent examination of the data and to a NIST analysis of selected samples from the batch for concentration value assignment.

Data that must be sent to NIST include:

1) cylinder fill date and method

2) manifold fill position with cylinders identified by cylinder and sample number

3) identification of batch standard

4) analytical data including

a. cylinder concentration

b. calibration standards

c. order of analysis

d. impurity data for specified species 
The data will be submitted to NIST via e-mail using the data submission form in Appendix. Word and Excel are the formats currently being used. Information can be obtained at gasntrm@nist.gov if there are any questions as to where the data should be submitted.

NIST will review the data to determine if:

1) the analytical method yields reliable results

2) the batch homogeneity meets NIST specifications

3) the batch concentration meets target values.

If the data supports it, NIST will proceed to the certification phase by selecting $10 \%$ of the batch (a minimum of 2 cylinders) for NIST evaluation. The batch standard will be included in the selected cylinders. The producer will then send to NIST these cylinders plainly labeled with sample number, producer, specie, and concentration. If the batch is rejected at this point due to evidence of heterogeneity in the submitted data, NIST may ask the producer to perform additional analytical work and resubmit the batch. However, if the heterogeneity is due to the fill method, the batch cannot be certified.

The correct address to use to ship cylinders to NIST can be obtained at gasntrm@ nist.gov

\section{BATCH CERTIFICATION}

\subsection{Overview}

After the producer submits the required data to NIST (see sec. 2.6), the data will be reviewed and $10 \%$ of the batch (a minimum of two cylinders) will be selected to be sent to NIST for analysis. Procedures that are similar to those used for SRM certification will be used to analyze the selected NTRM cylinders. The analyses will be used to determine the concentration of the analyte(s) and any impurities that may compromise the use of the NTRM. This process will normally be completed within three months of sample submission. A Report of Analysis, Certificates of Traceability, and cylinder labels will be prepared by NIST and issued to the producer for all approved NTRMs. A Report of Analysis will also be issued to the producer for rejected batches detailing the reasons the NTRM failed certification.

\subsection{NIST Analytical Protocol}

NIST analysis of the selected cylinders from the NTRM batch is modeled on the procedures used by NIST to analyze SRMs. In a typical NTRM certification procedure, the producer's batch standard is analyzed using a NIST reference method. Typically this method is an instrumental procedure calibrated with a gas reference material, such as gravimetrically prepared NIST primary standards, working standards, SRMs, or SRM lot standards. Then the same instrumental procedure is used to compare the remaining selected cylinders to the NTRM batch standard. The instrumentation used in these analyses is chosen based on: 1) a stable and noise free signal to ensure a high degree of precision in the intercomparisons; and, 2) an adequate signal resolution to detect small $(0.2 \%)$ concentration differences. The same instrumentation used for the certification of an SRM is used for the analysis of the corresponding NTRM. 
Since the concentration of an NTRM batch can be at or between NIST primary standard concentrations, two possible cases exist for the analysis of the selected cylinders. Where the NTRM and NIST primary standard concentration are close, an SRM, SRM lot standard, working standard, or primary standard will be used to analyze the producer's batch standard. If the concentration of the NTRM falls between NIST calibration standard concentrations, more standards will be used. The number and range of standards used to analyze this type of NTRM will depend on instrument function and the concentration of the NTRM.

In both cases, the producer's batch standard is analyzed by comparing it to a NIST standard(s) a minimum of six times on each of two different days. Potential instrument drift is corrected by alternately analyzing the NIST standard and the producer's batch standard. The NTRM response data is then correlated to the NIST standard response data using a linear or a quadratic instrument response function. Most of the instruments used by NIST have a linear response function.

After the producer's batch standard is analyzed, the instrumental procedure is used to compare the other selected cylinders to the batch standard. The same basic analytical sequence is followed, analyzing the producer's batch standard frequently to correct for instrument drift. A minimum of five comparisons of each sample to the producer's batch standard is made. The goal of the certification model is to minimize the measurement uncertainty.

\subsection{Producer's Data Validation and Batch Value Assignment}

The NIST concentration value for the producer's batch standard will be compared to the concentration determined by the producer. Since the producer is not responsible for final concentration value assignment, the producer's value need not be the same as that of NIST, but it should be close. A factor (f) is determined using the equation;

$$
f=C_{\mathrm{NIST}} / C_{\text {Producer }}
$$

where $C_{\mathrm{NIST}}$ is the concentration of the batch standard as determined by NIST, and $C_{\text {Producer }}$ is the concentration of the batch standard as determined by the producer. This factor is used to adjust the producer's analysis of the NTRM batch to the NIST analysis. If this factor is $1.000 \pm 0.005$, certification can proceed and the producer's data can be validated. If the factor deviates from unity more than $0.5 \%$ the certification may proceed but NIST will work with the producer to identify the reason for the deviation. If, after reconciliation between NIST and the producer, the factor still deviates from unity more than $0.5 \%$ the batch will be rejected.

NIST will also examine the data to determine if the ratio of the concentration values between the selected cylinders and the batch standard are the same as determined by both NIST and the producer. If the ratios determined by NIST are the same (within measurement uncertainty) as that determined by the producer, then the producer's data can be considered valid and certification can proceed. However, if the ratios do not match within measurement uncertainty the NTRM batch will be rejected.

Once the producer's data have been validated, NIST will examine the data for overall batch homogeneity. If the producer's data show that the batch is homogeneous and stable the batch can be certified. If the batch shows a percent relative standard deviation in the concentration of greater than $0.5 \%$, the batch will be rejected. A possible exception can be made where homogeneous batches are difficult to prepare due to the chemical nature of particular analytes. 
If the batch is found to be homogeneous, and the producer's data have been validated, then NIST will proceed to assign a concentration value. NIST first adjusts the producer's homogeneity data using factor $\mathrm{f}$ so each cylinder's concentration value reflects NIST's value assignment of the batch standard. The final certified concentration value for the batch is the average of the producer's NIST adjusted homogeneity data.

\subsection{Value Uncertainty}

The uncertainty [1] of the NTRM concentration value is estimated using a three-component additive analysis of variance model. The components are: 1) the assigned uncertainty of the standards used in the NIST analysis; 2) the measurement uncertainty of the NIST analysis of the NTRM batch standard; and, 3) the batch homogeneity measured by the producer.

A combined uncertainty for the NIST standard, $u_{\mathrm{PS}}$, is derived from the assigned $95 \%$ confidence interval of the standard $\left(U_{\mathrm{PS}}\right)$ using the appropriate coverage factor (example: an assigned $95 \%$ confidence interval of $1.5 \%$ would yield a combined uncertainty of $0.75 \%$, using a coverage factor of 2 ). A measurement combined uncertainty, $\mathrm{u}_{\mathrm{A}}$, is established from analytical experience and replicate measurements. A batch homogeneity combined uncertainty, $u_{\mathrm{H}}$, is established from the producer's analysis of all the cylinders in the batch.

This model is used when an NTRM that is close in concentration to a NIST primary standard and the concentration value assignment can be accomplished by direct comparison. In the case where the NTRM concentration falls between that of two NIST primary standards and its concentration is determined by interpolation, $u_{\mathrm{PS}}$ is larger than just the combined uncertainty of one primary standard since more than one standard is used in the analyses.

The combined uncertainty of the NTRM batch is estimated from the experimental combined uncertainties and is calculated using the following equation;

$$
u_{\mathrm{c}}=\left(u_{\mathrm{PS}}^{2}+u_{\mathrm{A}}^{2}+u_{\mathrm{H}}^{2}\right)
$$

The NTRM expanded uncertainty $U$ is calculated using the following equation;

$$
U=k u_{\mathrm{c}}
$$

with the coverage factor $\mathrm{k}$ being equal to 2 . The true concentration is asserted to lie within the interval defined by the batch certified value $\pm U$ with a level of confidence of approximately 95 percent [Ref. 1 ].

\subsection{NTRM Documentation}

After the certification process is completed, the NTRM cylinders are returned to the producer and NIST generates a Report of Analysis which is issued to the producer. The Report of Analysis contains:

1) the NIST-assigned NTRM batch concentration value and it's uncertainty

2) the certification period 
3) a description of the NIST value assignment procedure including the determination of the concentration factor(f)

4) a description of the NIST standard(s) used

5) a listing of the NTRM samples analyzed by NIST

6) NIST concentration values

7) producer's concentration values

8) a listing of all the certified cylinders in the NTRM batch

9) the concentration value for each as calculated by NIST.

A Certificate of Traceability is prepared by NIST for each NTRM batch. It displays the NTRM logo, the batch identification number, the concentration and uncertainty, and the certification period. A cylinder label for each cylinder is also prepared by NIST if requested by the producer. The labels also display the NTRM logo, the NTRM sample identification number, the NTRM cylinder number, the certification period and the batch concentration value and uncertainty.

Certification periods for non reactive mixtures is six years and for certain reactive gas mixtures it is four years. Mixtures of reactive gases such as $\mathrm{NO}, \mathrm{SO}_{2}$ and $\mathrm{H}_{2} \mathrm{~S}$ below $40 \mu \mathrm{mol} / \mathrm{mol}$ have certification periods of two years. NIST reserves the right to assign a specific certification period, different than stated here, to individual NTRM batches after evaluating data related to the NTRM batch. Any questions concerning certification periods should be addressed to gasntrm@nist.gov.

\subsection{NTRM Rejection Criteria}

NIST will reject an NTRM for any one of the following reasons:

1) impurity concentrations are too high

2) the concentration factor (f) diverges from unity more than $0.5 \%$ after reconciliation between NIST and the producer

3) the producer's and/or NIST's homogeneity data shows a concentration spread $>0.5 \%$ relative; and,

4) NIST's concentration ratios to the batch standard do not agree within measurement uncertainty with the producer's concentration ratios.

In all these cases NIST will work with the producer to resolve the problem for future NTRM work. NIST may reconsider the certification of a rejected batch if additional analytical work is performed by the producer and the batch resubmitted for certification.

\section{MAINTENANCE OF NTRM BATCHES}

\subsection{Batch Recertification}

An NTRM batch is certified from the date of the NIST Report of Analysis until an expiration date given in the report, defined as the certification period. When that time period expires, all cylinders comprising that NTRM batch are no longer certified. Customers who purchase an NTRM from a producer are constrained 
to this same expiration date. If the NTRM batch expires and subsequently loses certification, the customer's NTRM cylinder likewise loses certification. Thus it is very important that the producer maintains the NTRM batch certification status for the projected lifetime of the individual cylinder mixtures.

Batch recertification is the process the producer must follow in order to maintain certification of the NTRM. The purpose of this process is to verify batch concentration and to assure continuing stability of the gas mixture. To accomplish this, a cylinder(s) that is representative of the batch must be analyzed using a NIST certified standard. Two methods to accomplish this are as follows.

1) If the producer has retained the batch standard, and the batch standard was a member of the original batch production, then the batch standard may be analyzed as the representative of the batch; it is highly recommended that the BS be retained.

2) A minimum of two cylinders from the batch must be analyzed. If the producer no longer has the two required cylinders, due to distribution to customers, the cylinders may have to be recalled for analysis.

Also, it is recommended that the entire stock of cylinders from the batch that are still under the producer's control be analyzed.

Analysis of the batch standard, or the two representative cylinders, must be accomplished using the same procedure originally used by the producer. A NIST certified standard must be used to assign concentrations to these cylinders. The data generated by this analysis must then be sent to NIST. NIST will review the data and determine if the certification period should be extended. Usually if the data from the analysis match the original data within measurement uncertainty, or within half of the stated uncertainty from the original certified concentration, the certification period will be extended and a new certificate issued. NIST reserves the right to recall cylinders (usually the batch standard) from the producer for analysis by NIST. If the batch fails to be recertified, the producer must contact customers in possession of the NTRM cylinders. NIST reserves the right to request a listing of the disposition of all cylinders in the NTRM batch.

The recommended method of handling recertification of the NTRM batch is to analyze, prior to shipment, a customer's NTRM cylinder and the batch standard against a NIST certified standard. If the data continues to show good stability for the batch, e.g., the concentration is within half the stated uncertainty of the original certified value, the cylinder can be shipped. When the batch certification period expires, these data can be supplied to NIST as evidence for recertification. This approach is best used when the entire batch is expected to be sold or used prior to the expiration date. NIST will use the last analysis date as the basis for the extended certification period.

\subsection{Customer NTRM Cylinders}

A customer who purchases an NTRM cylinder from a producer receives a certificate with an expiration date identical to that of the batch. As outlined in section 5.1, the certification period is linked with the batch certification, which is the responsibility of the producer to maintain. The customer's certification expires at the end of the batch certification date. The customer must then contact the producer about recertification of their cylinder. 


\subsection{Customer NTRM Recertification}

If a producer agrees to perform recertification analyses for a customer's NTRM they should follow section 5.1.

If the concentration of the submitted NTRM is outside the original certified concentration uncertainty interval, recertification will be granted if there is a known cause for the discrepancy that NIST acknowledges, and it does not impact on the usability of the standard.

Once notified that the recertification is granted, the producer can issue to the customer written notification of the certification status. Certification will be extended for the current certification period from the date of the recertification report and an expiration given.

If a customer possesses an NTRM and the producer does not maintain certification then NIST should be contacted for discussion. NIST may agree to analyze individual NTRM cylinders for the extension of the certification period for that particular sample.

\subsection{Recertification of Non-original Producer's NTRM's}

If one company comes into the possession of an NTRM batch from a second company then it is the responsibility of the company that possesses the batch to maintain it. If two companies merge then it is the responsibility of the resultant company to maintain any NTRM batches that were assumed in the merge.

If a producer comes into possession of an NTRM Batch of another producer then recertification is not necessarily straightforward. The measurement system used for the original work may not be available. Recertification can be performed in one of two ways given below. However, NIST should be consulted before work is begun to assure that there is agreement as to the appropriateness of any action.

1) If the current owner of the Batch has a measurement system, including NIST traceable standards, identical to or similar to the original producer's then recertification can be accomplished using the regular procedure.

2) If a similar measurement system is not available then the current owner can use an appropriate instrument to compare the samples to the BS or other cylinder from the Batch. Provide the data to NIST for review and if the homogeneity is good, then, NIST will request the sample used as the control to be sent to NIST for analysis. The NIST value assignment of the selected cylinder along with the current owner's homogeneity data will be used for the determination of the analyzed sample concentrations. 


\section{TRACEABILITY}

\subsection{NTRM Batch Traceability}

The traceability of a gas NTRM batch relies on the unbroken chain of comparisons to NIST's measurement system which relies heavily on primary gas mixture standards. Through gravimetric preparation of the primary gas standards and international key comparisons, NIST assures traceability to the mole, a basic unit of the International System of Units, known as SI. Since the beginning of the NIST gas standards program, the NIST primary gas standards have been its foundation. They are prepared and maintained by NIST, and regularly undergo stringent internal evaluation and comparisons with other international gas standards. In the national hierarchy of gas standards, they are at the pinnacle. The NIST certified standards, such as gas SRMs, are compared to these primary gas standards for certification. These NIST-certified standards enable producers to prepare and maintain the NTRM batches that are provided to the public or used to produce other traceable gas standards.

There is an unbroken series of analyses comparing NTRM gas mixtures to NIST certified standards, and a direct NIST involvement in the NTRM certification. NIST maintains records of certified NTRMs and certification periods. If an NTRM batch is still in use after the certified period expires, it must be reanalyzed and the data submitted to NIST for concurrence concerning stability. NIST reserves the right to request any NTRM cylinder be sent to NIST for analysis if there are any questions concerning stability or concentration. The direct involvement of NIST in the quality assurance of each NTRM batch should provide continued acceptability of the certified concentrations with time.

\subsection{Research Gas Mixtures}

If traceable standards are required but NIST does not have existing primary standards or measurement system with defined uncertainty, then NIST can develop the required primaries or measurement system. The primaries can be distributed as a means for traceability or can be maintained at NIST to analyze other mixtures to be distributed. These mixtures that have no other pedigree but are certified by NIST are called Research Gas Mixtures (RGMs) and may be used for traceability purposes (Appendix 5).

\subsection{Mixtures Produced Referencing NTRMs and SRMs}

Not all of the analytical activities of gas analysis laboratories require the stringent traceability of an SRM or NTRM. In these instances a gas mixture further down the traceability chain may be all that is required. Gas mixtures prepared using a protocol referencing gas SRMs, NTRMs or RGMs may be declared as NIST traceable if the general guidelines given below are followed. It is important to realize that these guidelines represent the minimum required in order for NIST to recognize a gas mixture as traceable. The minimum requirements for a NIST traceable gas mixture are: 
1) The cylinders will be clean, of standard size and construction, and of known compatibility and history with the composition of the proposed standard. The cylinders will be equipped with valves of the appropriate material that conform to Compressed Gas Association (CGA) recommendations for the particular gas mixture. The reagent gases must be of high purity and quality to ensure that impurity levels and mixture stability meets NIST specifications.

2) The concentration of the mixture is within $5 \%$ relative of the SRM, NTRM or RGM concentration. The composition of the mixture should be identical to that of the SRM or NTRM. Multicomponent mixtures may be traceable to binary mixtures as long as there are no component interactions, and the balance gas is identical to the referenced mixtures (or chemically inert to the components). Stability must be assured through procedures similar to section 3.5 or demonstrated by data available to customers.

3) All sources of uncertainty in the concentration assignment are included in the final uncertainty statement. The model given in section 4.4 should be followed. The combined uncertainty of the referenced SRM, NTRM or RGM must be included in the mixtures expanded uncertainty.

4) An expiration date for the mixture must be given to the customer. The period of time the standard is valid will not exceed the original certification period of the corresponding SRM, NTRM or RGM. Example: If the original NTRM was certified for 2 years, the mixture can be given a maximum validation period of 2 years.

5) Documentation provided the customer will include concentration, expanded uncertainty, expiration date, significant impurities, and the specific SRM, NTRM or RGM used for concentration value assignment.

An excellent document describing a method to prepare a gas mixture referencing an SRM, NTRM or RGM is given in reference 3 .

\section{References}

1) Joint Committee for Guides in Metrology: BIPM, Sevres Cedex, France (2008), JCGM 100:2008, Guide to the Expression of Uncertainty in Measurement. See also B. N. Taylor and C. E Kuyatt, NIST Technical Note 1297, 1994 Edition, Guidelines for Evaluating and Expressing the Uncertainty of NIST Measurement Results,

2) U.S. Environmental Protection Agency, EPA-600/R93/224, September 1993, EPA Traceability Protocol for Assay and Certification of Gaseous Calibration Standards.

3) E. E. Hughes and J. Mandel, NBSIR 81-2227 (revised 1989), A Procedure for Establishing Traceability of Gas Mixtures to Certain National Institute of Standards and Technology SRM's.

4) International vocabulary of basic and general terms in metrology (VIM), 1993, issued by BIPM, IEC, ISO, IUPAC, IUPAP and OIML. 
5) ISO Guide 30: 1981, Terms and definitions used in connection with reference materials. See also ISO 8402, Quality - Vocabulary, 1986.

6) J. Phys. Chem. Ref. Data, Vol. 34, No. 1, 2005 p.61, Isotopic Composition of the Elements, 2001.

\title{
Contact Information and Websites
}

\author{
NTRM Contact \\ ntrm@nist.gov \\ NIST Home Page \\ http://www.nist.gov \\ SRM Home Page \\ http://www.nist.gov \\ select "A-Z Index"; select "Standard Reference Materials" \\ Original NTRM Document (including Reporting Forms) \\ http://www.nist.gov/srm/upload/SP260-126.PDF \\ Guide to the Expression of Uncertainty \\ http://www.bipm.org/utils/common/documents/jcgm/JCGM_100_2008_E.pdf
}




\section{Appendix 1: Definition of Terms}

This section provides definitions of terms used in this document. The initial use of each of these terms within this document is signified by emphasized print. These terms may be defined differently in other documents released by NIST.

Batch: A group of gas mixtures, contained in gas cylinders, prepared by a producer in such a manner that all the gas mixtures are identical in composition and concentration. For the purposes of this program, each batch will contain a minimum of 10 cylinders.

Batch Standard: A gas mixture, similar in concentration and uncertainty to the NTRM batch, to which all cylinders of the batch are related through chemical analysis. The batch standard is usually a randomly chosen member of the NTRM batch. If the batch standard is not a member of the NTRM batch, its concentration must be within $5 \%$ of the batch nominal concentration.

Calibration Standards: Gas mixture standards that include primary gravimetric standards, SRMs, SRM Lot Standards, Working Standards, permeation tubes, or other means to introduce an analyte into a diluent gas. These are all characterized and maintained at NIST and the values assigned are included in Reports of Analysis with the uncertainties explained and quantified.

Certification Period: The period of time a gas mixture is certified for component concentration.

Concentration: The amount-of-substance (SI unit: mole) of the subject compound within the cylinder. This is usually expressed as the amount-of-substance fraction (SI unit: $\mathrm{mol} / \mathrm{mol}$ ).

Heterogenous: Having a concentration spread in excess of $0.5 \%$ relative standard deviation.

Homogeneity: A measure of the concentration spread exhibited by an NTRM batch. Usually measured as a percent relative standard deviation from the average concentration.

Homogeneous: Having a concentration spread of less than $0.5 \%$ relative standard deviation.

Impurities: Unwanted chemical compounds within a gas mixture. Their concentration must not be large enough to jeopardize the intended use of the mixture.

Incubation Period: The period of time after production the gas mixtures are required to mature before analyses are performed. This period allows the gas mixture to stabilize to their final concentration, giving a better measure of batch homogeneity.

Instrument Response Function: The relationship of instrument response to analyte concentration. Determine whether it fits a linear or non-linear function.

Measurement System: The means by which NIST will perform certification analyses; includes calibration standards and instrumentation. 
NIST-Certified Standard: Gas mixtures that have been certified by NIST and which are within the valid certification period. Documentation in the form of a NIST Report of Analysis or Certificate must be available for inspection. An SRM is a valid traceable standard that can be used in NTRM production (note that Research Gas Mixtures (RGMs) should be approved by NIST before use in NTRM production.

NIST Primary Standards: Gas mixtures maintained by NIST, which have been prepared gravimetrically at NIST, to which all measurements are ultimately compared.

NIST Traceability: Traceability is to SI units. However NIST recognizes that "Traceability to NIST" is present in regulation to define standards to be used to satisfy regulatory requirements. Therefore NIST provides documentation for NTRMs and RGMs which reference this term.

NIST-Traceable Reference Material (NTRM): A reference material produced by a commercial supplier with a well-defined traceability linkage to the National Institute of Standards and Technology (NIST). This linkage is established via criteria and protocols defined by NIST that are tailored to meet the needs of the metrological community to be served. Reference material producers adhering to these requirements will be allowed the "NTRM" Certification Mark.

NTRM Prime: Prepared and analyzed in the same manner as a normal NTRM but after the producer finishes the analytical work, every cylinder in the Batch is submitted to NIST for analysis and an individual value and uncertainty placed on each cylinder.

Quality System: The organizational structure, responsibilities, procedures, processes and resources for implementing quality management.

Recertification: A process that must be completed in order for the gas mixtures certification period to be extended.

Research Gas Mixture (RGM): A gas mixture that is either prepared or analyzed by NIST and has no other pedigree. This may be prepared gravimetrically with known composition from the blending or analyzed at NIST using a measurement system with defined uncertainty. A report of Analysis describing the preparation and/or analysis must be prepared and maintained in NIST records.

Resolution: An instrument's ability to detect small changes in signal and report this change in the output data stream.

Sample Number: A sequential number assigned to the cylinders of an NTRM. The sample numbers span from 1 through the number of cylinders in the batch. They are permanently associated with the cylinders throughout the lifetime of the NTRM.

Selected Cylinders: Cylinders from the NTRM batch that are selected by NIST to be sent by the producer to NIST for value assignment.

Sensitivity: An instrument's ability to distinguish a true analytical signal from background noise.

SRM Lot Standard: The standard used by NIST to certify an SRM lot. The Lot Standard is also used to track the stability of the SRM lot. 
Stability: A measure of the concentration drift of a gas mixture over time. A gas mixture is stable if the concentration drift is small relative to the absolute concentration over time. For NTRMs the concentration drift must be within half of the stated uncertainty from the certified concentration over the entire certification period.

Standard Reference Material (SRM): Certified reference materials issued by NIST. These are wellcharacterized materials produced in quantity to improve measurement science. SRMs are certified for specific chemical or physical properties, and are issued by NIST with certificates that report the results of the characterization and indicate the intended use of the material.

Traceability: The property of a result of a measurement whereby it can be related to appropriate standards, generally national or international standards, through an unbroken chain of comparisons [Ref. 4,5].

Uncertainty: A parameter, associated with concentration, which characterizes the dispersion of the values that attributed to the concentration assignment. A reported concentration value must have an associated uncertainty [Ref. 1,4,5].

Working Standard: A gas mixture standard that has been value-assigned using a NIST measurement system with defined uncertainty. 


\section{Appendix 2: Production and Certification Flow Chart with Referenced Sections}

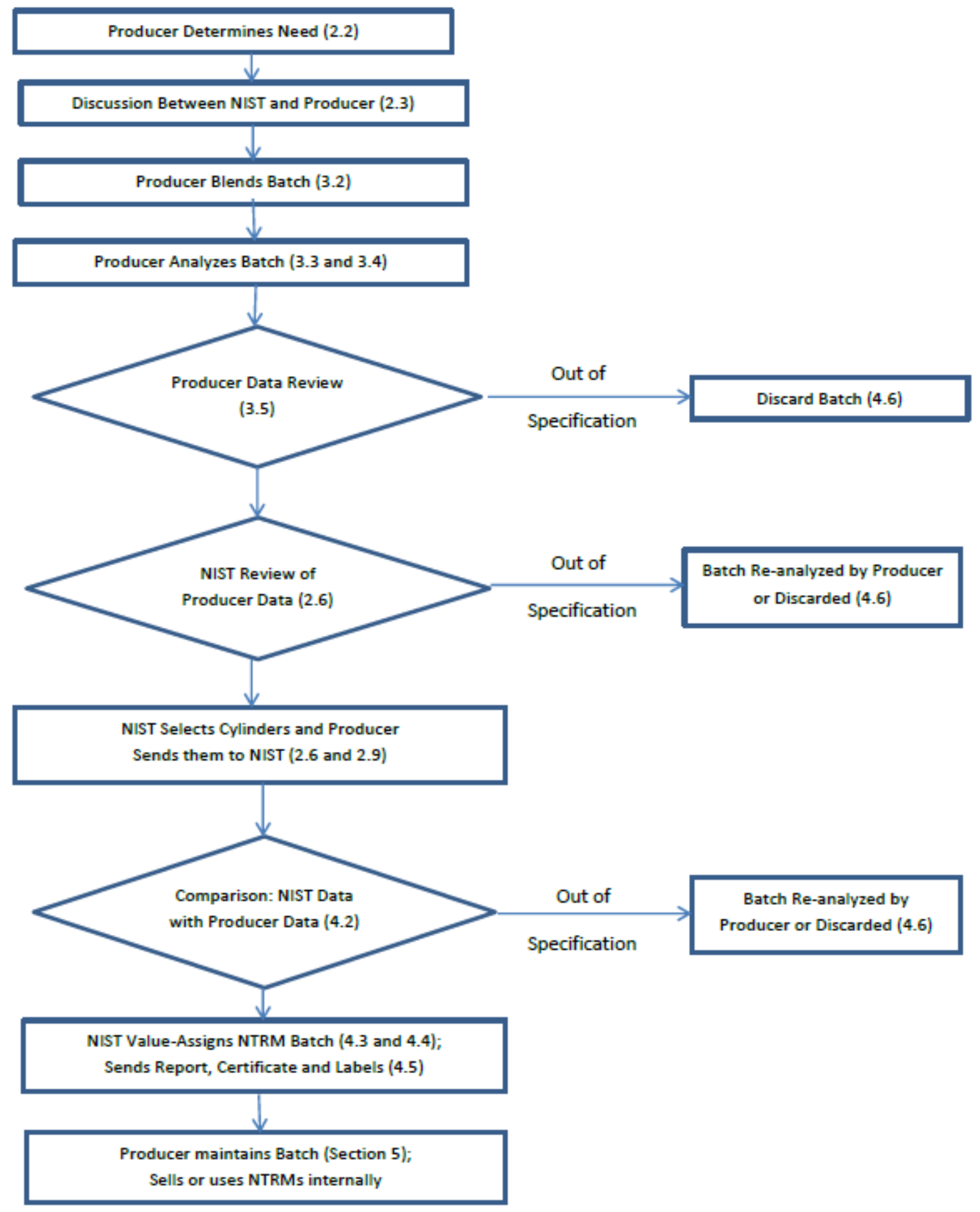




\section{Appendix 3: NTRM Specifications}

\section{A partial listing of some primary gas standard suites which may bound NTRMs:}

\author{
$\underline{\text { Mixture Components }}$ \\ Carbon Monoxide in Air \\ Carbon Monoxide in Nitrogen \\ Carbon Dioxide in Air \\ Carbon Dioxide in Nitrogen \\ Propane in Air \\ Propane in Nitrogen \\ Methane in Air \\ Methane in Nitrogen \\ Oxygen in Nitrogen \\ Ethanol in Nitrogen \\ Nitric Oxide in Nitrogen \\ Sulfur Dioxide in Nitrogen \\ Hydrogen Sulfide in Nitrogen \\ Volatile Organics \\ Natural Gas \\ Any other mixtures
}

Amount of Substance Range
$50 \mathrm{nmol} / \mathrm{mol}$ to $500 \mathrm{nmol} / \mathrm{mol}$
$10 \mu \mathrm{mol} / \mathrm{mol}$ to $45 \mu \mathrm{mol} / \mathrm{mol}$
$10 \mu \mathrm{mol} / \mathrm{mol}$ to $15 \% \mathrm{~mol} / \mathrm{mol}$
$360 \mu \mathrm{mol} / \mathrm{mol}$ to $420 \mu \mathrm{mol} / \mathrm{mol}$
$5 \mu \mathrm{mol} / \mathrm{mol}$ to $20 \% \mathrm{~mol} / \mathrm{mol}$
$0.1 \mu \mathrm{mol} / \mathrm{mol}$ to $500 \mu \mathrm{mol} / \mathrm{mol}$
$5 \mathrm{nmol} / \mathrm{mol}$ to $2 \% \mathrm{~mol} / \mathrm{mol}$
$1 \mu \mathrm{mol} / \mathrm{mol}$ to $1000 \mu \mathrm{mol} / \mathrm{mol}$
$0.5 \mu \mathrm{mol} / \mathrm{mol}$ to $10 \% \mathrm{~mol} / \mathrm{mol}$
$0.4 \% \mathrm{~mol} / \mathrm{mol}$ to $25 \% \mathrm{~mol} / \mathrm{mol}$
$50 \mu \mathrm{mol} / \mathrm{mol}$ to $500 \mu \mathrm{mol} / \mathrm{mol}$
$0.5 \mu \mathrm{mol} / \mathrm{mol}$ to $3000 \mu \mathrm{mol} / \mathrm{mol}$
$5 \mu \mathrm{mol} / \mathrm{mol}$ to $5000 \mu \mathrm{mol} / \mathrm{mol}$
$1 \mu \mathrm{mol} / \mathrm{mol}$ to $500 \mu \mathrm{mol} / \mathrm{mol}$
Contact NIST
Contact NIST
Contact NIST

This list is not complete, any questions concerning other constituents and/or concentrations can be answered by contacting NIST at gasntrm@nist.gov.

\section{Minimum incubation period before first analysis:}

Two days for $\mathrm{CO}, \mathrm{CO}_{2}, \mathrm{C}_{3} \mathrm{H}_{8}, \mathrm{CH}_{4}$ and $\mathrm{O}_{2}$ then 30 days between the first and second analyses.

Four days for $\mathrm{NO}$ and $\mathrm{SO}_{2}$ then 30 days between the first and second analyses.

For reactive mixtures below $50 \mu \mathrm{mol} / \mathrm{mol}$ a minimum of 3 months between analyses is required

Maximum allowable impurity levels: Impurity levels are the same as those stipulated for gas mixture SRMs and these are periodically updated on the NIST SRM web site. If there are any questions concerning impurity levels, the above stated web site will address them. If there are any questions contact NIST at gasntrm@nist.gov.

Isotopic Distribution: The carbon monoxide and carbon dioxide analytes in mixtures must have the natural distribution of ${ }^{12} \mathrm{C}$ and ${ }^{13} \mathrm{C} ;{ }^{12} \mathrm{C}=98.85 \%-99.04 \%$ and ${ }^{13} \mathrm{C}=1.15 \%-0.96 \%$ [6].

\section{Carbon Monoxide Mixtures}
Water vapor
$1 \mu \mathrm{mol} / \mathrm{mol}$
Carbon dioxide
$1 \mu \mathrm{mol} / \mathrm{mol}$ or $0.1 \%$ of the $\mathrm{CO}$ value whichever is greater 
Methane

Other Hydrocarbons

\section{Carbon Dioxide Mixtures}

Water vapor

Carbon monoxide

Nitrous oxide

Hydrocarbons

Propane Mixtures

Water vapor

Other hydrocarbons

Methane Mixtures

Water vapor

Other hydrocarbons

\section{Oxygen Mixtures}

Water vapor

Hydrocarbons as methane

Argon in $2 \% \mathrm{~mol} / \mathrm{mol}$ oxygen

Argon in $10 \% \mathrm{~mol} / \mathrm{mol}$ oxygen

Argon in $21 \% \mathrm{~mol} / \mathrm{mol}$ oxygen

Total other impurities

\section{Nitric Oxide Mixtures}

Water vapor

Nitrogen Dioxide

Hydrocarbons

$\underline{\text { Sulfur Dioxide Mixtures }}$

Water vapor

Hydrocarbons
$0.1 \%$ of the $\mathrm{CO}$ value

$0.1 \mu \mathrm{mol} / \mathrm{mol}$ as methane

$1 \mu \mathrm{mol} / \mathrm{mol}$

$0.01 \%$ of $\mathrm{CO}_{2}$ value

$0.1 \%$ of $\mathrm{CO}_{2}$ value

$0.1 \mu \mathrm{mol} / \mathrm{mol}$ as methane

$1 \mu \mathrm{mol} / \mathrm{mol}$

$0.4 \%$ of the propane value expressed as propane

$1 \mu \mathrm{mol} / \mathrm{mol}$

$0.5 \%$ of methane value

$1 \mu \mathrm{mol} / \mathrm{mol}$

$3 \mu \mathrm{mol} / \mathrm{mol}$

$30 \mu \mathrm{mol} / \mathrm{mol}$

$50 \mu \mathrm{mol} / \mathrm{mol}$

$100 \mu \mathrm{mol} / \mathrm{mol}$

$15 \mu \mathrm{mol} / \mathrm{mol}$

$1 \mu \mathrm{mol} / \mathrm{mol}$

$1 \%$ of NO value (all batch cylinders analyzed)

$2 \mu \mathrm{mol} / \mathrm{mol}$ expressed as methane

$1 \mu \mathrm{mol} / \mathrm{mol}$

$2 \mu \mathrm{mol} / \mathrm{mol}$ expressed as methane 


\section{Appendix 4: Data Submission Forms for Certification and Recertification Data}

The following form may be used to report the producer's NTRM information and analysis results to NIST. This form is available from NIST via e-mail or from the website (p.21).

FORM A - Producer's Report of Analysis of Candidate NTRM

$\begin{array}{ll}\text { Prepared by: } & \text { Report Date: } \\ & \text { Company Name: } \\ & \text { Address: } \\ & \text { Individual preparing report: } \\ & \text { Phone } \\ & \text { FAX } \\ & \text { e-mail }\end{array}$

Description: NTRM Composition:

Corresponding SRM No.:

Date Blended:

Blending Location:

(Address, Phone, FAX and Contact Person)

Analytical Location:

(Address, Phone, FAX and Contact Person)

Blending Method:

Manifold fill position diagram attached upon request:

Cylinders:

Size:

Material:

Manufacturer:

Treatment Process:

Previous Use:

Valve:

CGA \#:

Straight or Tapered Threads:

Manufacturer: 
Form A (continued)

Standards Used as Calibrants:

\begin{tabular}{llll} 
& & (If SRM) & $\begin{array}{l}\text { Concentration } \\
\text { No. }\end{array}$ \\
\hline 1. & Cylinder No. & Sample No. & \\
2. & & & \\
3. & & & \\
4. & &
\end{tabular}

Brief Description of Analytical Method:

Instrument Calibration Procedure:

Calibration Date:

Analytical Sequence (order in which cylinders were analyzed):

$\begin{array}{lll}\text { No. Calibrant } & \text { Concentration } & \begin{array}{l}\text { Instrument } \\ \text { Response }\end{array} \\ 1 . & & \\ 2 . & & \\ 3 . & & \\ 4 . & & \\ 5 . & & \\ \text { etc. } & \end{array}$

Analysis of NTRM Batch Standard:

Analysis Date:

Batch Standard Description:

Analytical Sequence (order in which cylinders were analyzed):

\begin{tabular}{|c|c|c|c|}
\hline & Cylinder or & Instrument & Calculated \\
\hline$\frac{\text { No. }}{1}$ & $\underline{\text { Calibrant }}$ & $\underline{\text { Response }}$ & Concentration \\
\hline
\end{tabular}

$\begin{array}{ll}\text { Average } & = \\ \text { Std. Dev. } & = \\ \% \text { Relative SD } & =\end{array}$


Form A (continued)

Analysis of NTRM Cylinders against Batch Standard:

Date:

Analytical Sequence (order in which cylinders were analyzed)

$\begin{array}{lll}\text { Sample } & \begin{array}{l}\text { Cylinder } \\ \text { Number }\end{array} \\ \frac{\text { No. }}{1} & & \\ 2 & & \\ \cdot & & \\ 20 & & \\ \text { etc. } & \text { Average } & = \\ & \text { Std. Dev. } & = \\ & \text { \% Relative SD }=\end{array}$

Impurity Analyses:

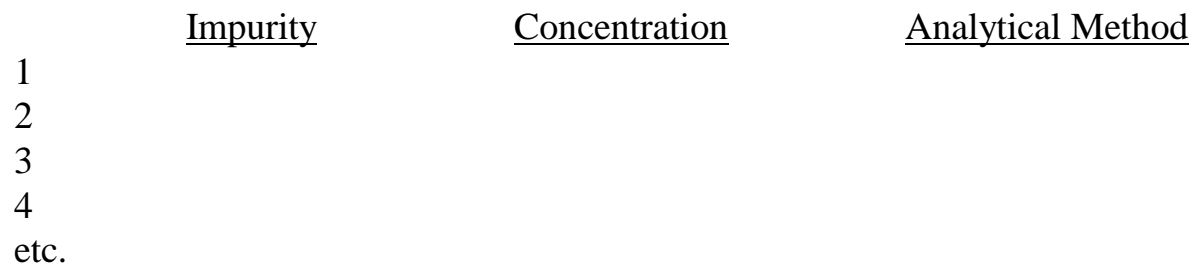


FORM B - Producer's Report of Analysis for Recertification of an NTRM

$\begin{array}{ll}\text { Prepared by: } & \begin{array}{l}\text { Report Date: } \\ \text { Company Name: } \\ \text { Address: }\end{array} \\ & \text { Individual preparing report: } \\ & \text { Phone } \\ & \text { FAX } \\ & \text { e-mail } \\ & \text { Location of Recertification Analyses }\end{array}$

Description: NTRM Composition:

Corresponding SRM No.:

Date Blended:

NIST Reports: $\quad$ Original Certification Report \#

Recertification Report \#s

$\underline{\text { Standards Used as Calibrants for Recertification: }}$

$\begin{array}{llll}\text { No. } & \text { Cylinder No. } & \begin{array}{l}\text { (If SRM) } \\ \text { Sample No. }\end{array} & \begin{array}{l}\text { Concentration } \\ \text { and uncertainty }\end{array}\end{array}$

1.

2.

3.

4.

Brief Description of Analytical Method:

Instrument Calibration Procedure:

Calibration Date:

Analytical Sequence (order in which cylinders were analyzed):

$\begin{array}{lll}\text { No. Calibrant } & \text { Concentration } & \begin{array}{l}\text { Instrument } \\ \text { Response }\end{array} \\ 1 . & & \\ 2 . & & \\ \text { etc. } & & \end{array}$


Analysis of NTRM Batch Standard:

Analysis Date:

Batch Standard Description:

Analytical Sequence (order in which cylinders were analyzed):

$\begin{array}{llll}\text { No. } & \begin{array}{l}\text { Cylinder or } \\ \text { Calibrant }\end{array} & \begin{array}{l}\text { Instrument } \\ \text { Response }\end{array} & \begin{array}{l}\text { Calculated } \\ \text { Concentration }\end{array} \\ 2 & & & \\ \text { etc. } & & & \end{array}$

$\begin{array}{ll}\text { Average } & = \\ \text { Std. Dev. } & = \\ \% \text { Relative SD } & =\end{array}$

Analysis of NTRM Cylinders against Batch Standard:

Date:

Analytical Sequence (order in which cylinders were analyzed)

$\begin{array}{lll}\begin{array}{l}\text { Sample } \\ \text { No. }\end{array} & \begin{array}{l}\text { Cylinder } \\ \text { Number }\end{array} \\ 2 & & \\ \cdot & & \\ . & & \\ 20 & & \text { Average } \\ \text { etc. } & \text { Std. Dev. } & = \\ & & \text { \% Relative SD }=\end{array}$




\section{Appendix 5: Research Gas Mixtures (RGM)}

\subsection{Back Ground}

The two major gas standards programs by which traceability to NIST is accomplished are the SRM and the NTRM programs. Both programs have as their foundation NIST primary standards or a NIST measurement system with defined uncertainty. If traceable standards are required but NIST does not have existing primary standards, then NIST can develop the required primaries. The primary standards developed under this program can be used by NIST to support a new SRM or a new NTRM or special analysis mixtures. The special analysis mixtures would be called Research Gas Mixtures (RGMs) and would not have the lot or batch size specifications, therefore they could be provided as fewer cylinders. Standards produced under this program can be initiated by NIST, other government organizations or private companies.

\subsection{Traceability}

Compressed gas mixtures containing environmentally and industrially important compounds have been certified by the National Institute of Standards and Technology (NIST) for over thirty years. These gas mixtures are analyzed by NIST for compound concentration and uncertainty expressed as the amount-ofsubstance fraction (concentration). The concentration value is directly traceable to units of mass and moles; both SI units. NIST gravimetrically-prepared primary standard gas mixtures (PSMs) and other internal mass based calibrations facilitate the vertical linkage which directly links the gas mixture concentration assignment through measurements to SI unit. A Technical Procedure (TP) [839.03.11E] exists within the NIST quality system and it presents the general procedures and methods used by NIST to analyze compressed gas mixtures which do not fall under the SRM or NTRM programs. When these gas mixtures are certified or prepared by NIST, they become Research Gas Mixtures (RGM), and the analytical work is documented in a Report of Analysis which serves as the certificate for the owner of the gas mixture.

\subsection{Scope}

The TP describes a general analytical scheme used to analyze gas mixtures not covered under the SRM or NTRM programs. Since these gas mixtures are mainly used in research, and are usually composed of components and/or concentrations that NIST has little experience, certification may be based on specialized analytical methods which have no previous documentation. Therefore the actual analytical methods used in the certification shall be documented in the analyst's notebook and Report of Analysis. Certification may be granted for a limited time period over which NIST has confidence that the gas mixture will remain stable. This time period may be a little as one month to as much as 8 years, or could be on an "as analyzed" basis with no certification granted. This depends on the stability information that NIST has obtained, and of the intended use of the gas mixture. 